\title{
Archéologie, art contemporain et recyclage des déchets
}

Archaeology, contemporary art and recycling waste

Jean Paul Demoule

\section{(2) OpenEdition}

1 Journals

Édition électronique

URL : https://journals.openedition.org/tc/6321

DOI : $10.4000 /$ tc. 6321

ISBN : 1952-420X

ISSN : 1952-420X

Éditeur

Éditions de l'EHESS

Édition imprimée

Date de publication : 15 juin 2012

Pagination : 160-177

ISBN : 2-7351-1512-7

ISSN : 0248-6016

Référence électronique

Jean Paul Demoule, "Archéologie, art contemporain et recyclage des déchets », Techniques \& Culture [En ligne], 58 | 2012, mis en ligne le 17 décembre 2012, consulté le 29 septembre 2022. URL : http:// journals.openedition.org/tc/6321 ; DOI : https://doi.org/10.4000/tc.6321

Tous droits réservés 


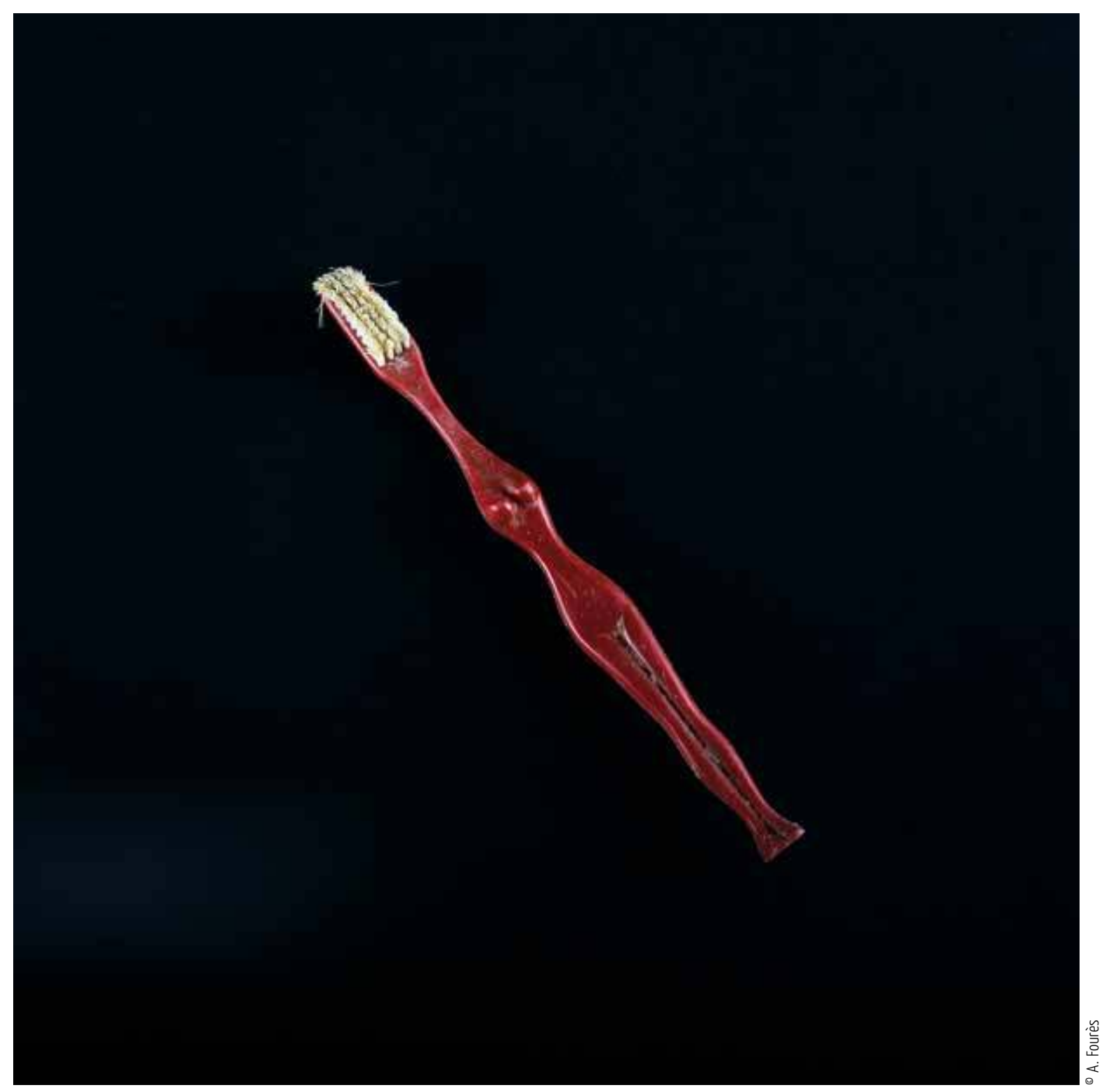




\section{ARCHÉOLOGIE, ART CONTEMPORAIN ET RECYCLAGE DES DÉCHETS}

Il y a diverses catégories d'objets irremplaçables, mais j'en fréquente au moins deux, les objets archéologiques d'une part, et les objets d'art de l'autre - deux catégories qui dans les premiers temps de l'archéologie ne faisaient qu'une. Et si l'on se replace même dans une durée encore plus longue, depuis qu'existent des objets que nous nommons « d'art », ceux-ci étaient sans doute chargés d'une valeur importante, bien au-delà de leur simple attrait esthétique, si l'on pense aux fameuses « Venus » de la préhistoire, dont les plus anciennes remontent à 35000 ans. Celle de Hohle Fels par exemple, en ivoire et trouvée dans une grotte du Jura souabe, serait la plus ancienne de toutes: si ses caractères sexuels, primaires et secondaires, sont largement exagérés, elle n'a intentionnellement pas de tête, laquelle est remplacée par un petit anneau. Elle n'était certainement pas confinée à un rôle décoratif mais était déjà un signe et un symbole, de fertilité et de fécondité comme on le répète usuellement - ou plutôt un objet pour penser la sexualité, d'un point de vue masculin, pour la seule espèce biologique dont la sexualité ne soit pas soumise à des interruptions cycliques dans son fonctionnement.

Ces premiers objets d'« art » sont irremplaçables ne serait-ce que par leur rareté, et tant qu'ils témoignent, en dépit de la vision du monde certainement partagée dont ils sont le symptôme, de gestes plastiques à chaque fois singuliers. D'une manière générale, ce sera l'une des caractéristiques des œeuvres d'art jusqu'à nos jours. C'est lorsque de tels objets commencent à être produits en série, comme les statuettes grecques dites «Tanagra » (une ville de Béotie), dans les derniers siècles avant notre ère, que l'on peut parler d'objets remplaçables et que l'on sort de ce que nous appelons actuellement « art »: ces statuettes votives sont moulées pour être fabriquées en série. Une chaîne discontinue
À la mémoire de Sandrine Henry-Duplessis 


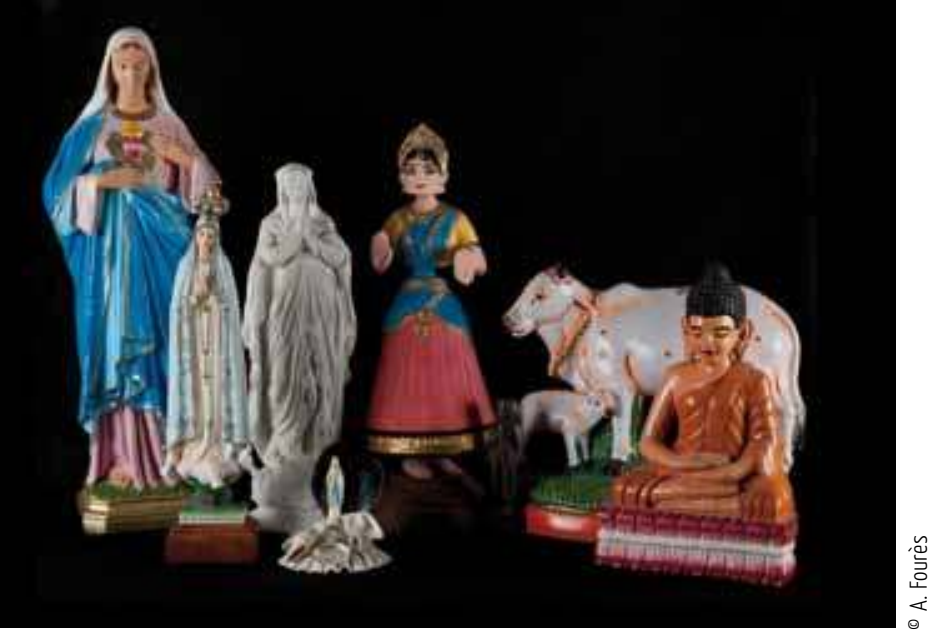

Objets de piété figuratifs liés à diverses religions contemporaines et fabriqués en série: objets d’art, ou objets kitsch? (Fig. 1)

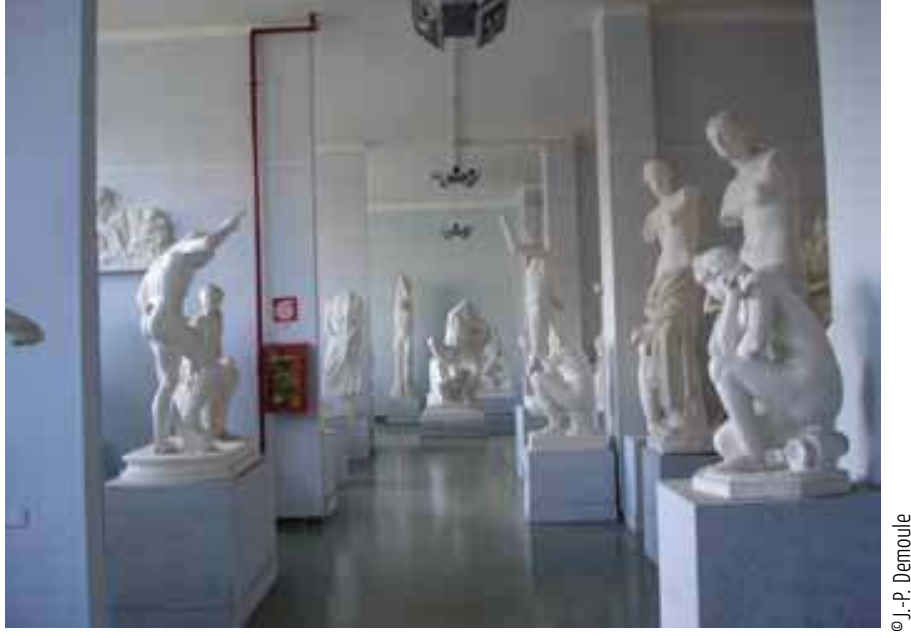

Quelques-uns des moulages en plâtre des chefs-d'œuvre de l'Antiquité gréco-romaine tels quilis sont exposés dans les galeries de l'Université La Sapienza à Rome. (Fig. 2)

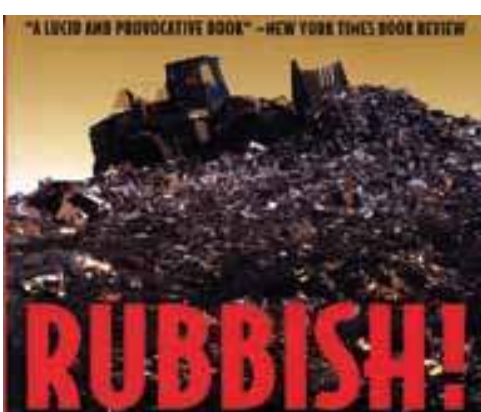

\section{THE ARCHAEOLOGY OF GARBAGE}
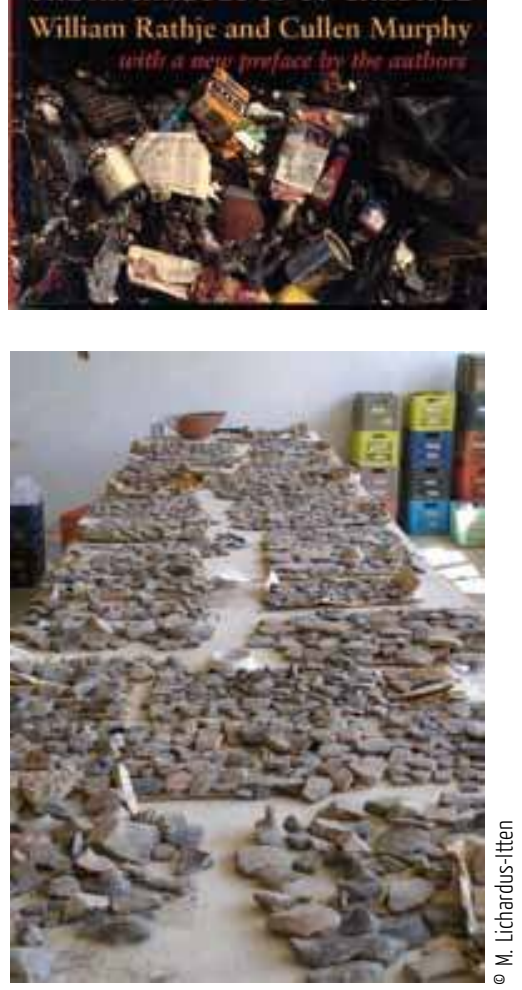

L'ouvrage de William Rathje et Cullen Murphy, Rubbish! The Archaeology of Garbage (1992), a inauguré dès les années 1970 la fouille des détritus contemporains, en montrant leur valeur heuristique pour la compréhension de nos sociétés.

(Fig. 4)

Table d'étude de quelquesuns des 1,7 million de tessons de poterie sur le site néolithique de Kovacevo, Bulgarie: qu'en faire après analyse?

(Fig. 5) les relie par exemple aux statuettes mariales que l'on peut acquérir de nos jours à Lourdes ou bien dans des sanctuaires catholiques - et elles ont leur équivalent dans beaucoup d'autres religions (Figure 1). Peut-être s'il n'avait surnagé des siècles passés qu'une seule statuette de Tanagra, et non des milliers, nous situerions-nous alors dans l'irremplaçabilité, et donc dans l'art. Symétriquement, comme on le rappellera plus bas, la problématique des ready made de Duchamp et de ses émules a procédé justement de la remise en cause du statut de l'art en qualifiant d'« artistiques » des objets fabriqués en série.

Le « beau » n'est donc pas en cause à ce stade, mais seulement la remplaçabilité, qui montre ici son caractère opératoire dans la logique du présent volume. Il en est de même de tout objet archéologique, d'« art » ou non, encore que la perception des archéologues n'a cessé d'évoluer. Seuls à la Renaissance relevaient de l'archéologie les objets d'« art » (Figure 2). Puis au cours du $\mathrm{XIX}^{\mathrm{e}}$ siècle, dans une trajectoire qui n'est pas partout achevée, l'archéologie est devenue l'approche anthropologique des sociétés à travers leurs objets, quelle que soit l'ancienneté de la société étudiée (Figure 3), au point que l'on pratique désormais une archéologie non triviale des sociétés contemporaines, telle l'« archéologie des poubelles » ou garbology, de l'archéologue américain Rathje (Figure 4). Pendant longtemps pourtant, on a jeté (et on jette encore sur certaines fouilles) tout objet « remplaçable », au sens où il serait d'une valeur insuffisante dans un rapport entre le coût de son traitement et de sa conservation d'une part, et sa valeur en terme de connaissance de l'autre. On jette cependant de moins en moins d'objets sur une fouille, même si l'on considère actuellement que plus une fouille visera à l'exhaustivité, plus elle sera coûteuse, le coût s'accroissant de plus en plus, à mesure que des informations supplémentaires sont gagnées, elles-mêmes de moins en moins importantes (Figure 5). D'ailleurs l'engorgement par les objets est tel que l'on doit, d'une manière plus ou moins revendiquée ou 


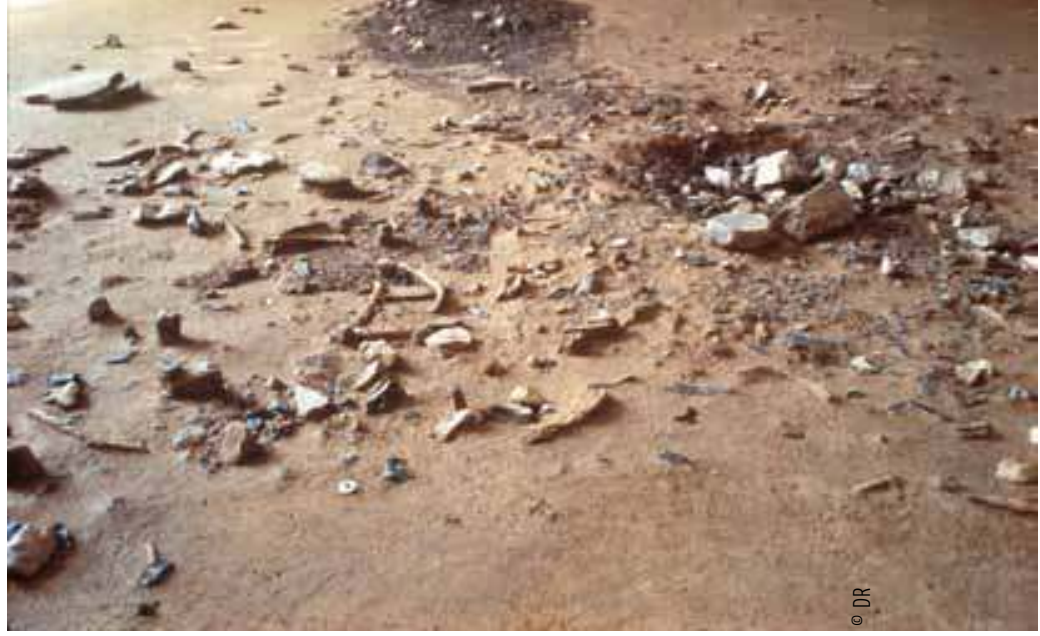

La fouille du site paléolithique de Pincevent (ici l'unité J116), qui a marqué une date dans l'approche anthropologique des sociétés préhistoriques. (Fig. 3)

plus ou moins sournoise, se défaire régulièrement de telles ou telles caisses d'échantillons ou de débris archéologiques très pauvres en informations, voire sans provenance désormais connue. Cela ne concerne cependant que le stockage antérieur à l'inventorisation dans un musée, car les collections des musées sont en principe «inaliénables », c'est-à-dire qu'on ne peut juridiquement s'en défaire.

\section{Du sexe au pouvoir}

Pendant tout le paléolithique et une partie du néolithique, les objets « d'art » représentatifs continueront à montrer des femmes nues et des animaux, et serviront donc à penser en ces termes la société (Figures 6 et 7). À côté de ces objets chargés de sens, on observe aussi une esthétique du quotidien, pour reprendre une distinction qui remonte à Franz Boas (1927). Il faudrait également faire la différence entre ce qui est représentatif et ce qui ne l'est pas - comme les signes abstraits gravés ou peints sur les parois des grottes; mais aussi entre ce qui serait simplement ornemental (figuratif ou non), et ce qui serait fonctionnel, soit dans le champ du matériel (actions sur la matière), soit dans celui du symbolique (comme les statuettes de femmes nues).

Au cours du néolithique, avec les premiers symptômes de différenciations et de hiérarchisations sociales, de nouvelles représentations apparaissent, celle du

\section{Figurine féminine}

Elle provient du site néolithique de Kovacevo, Bulgarie, fin du VIle millénaire. II s'agit comme au paléolithique du thème privilégié de représentation humaine.

(Fig. 6)

\section{Figurines animales}

Elles proviennent du site néolithique de Kovacevo, Bulgarie, fin du Vlle millénaire. Ces représentations, beaucoup moins nombreuses et beaucoup moins soignées que les figurines féminines, ont sans doute une autre fonction.

(Fig. 7)

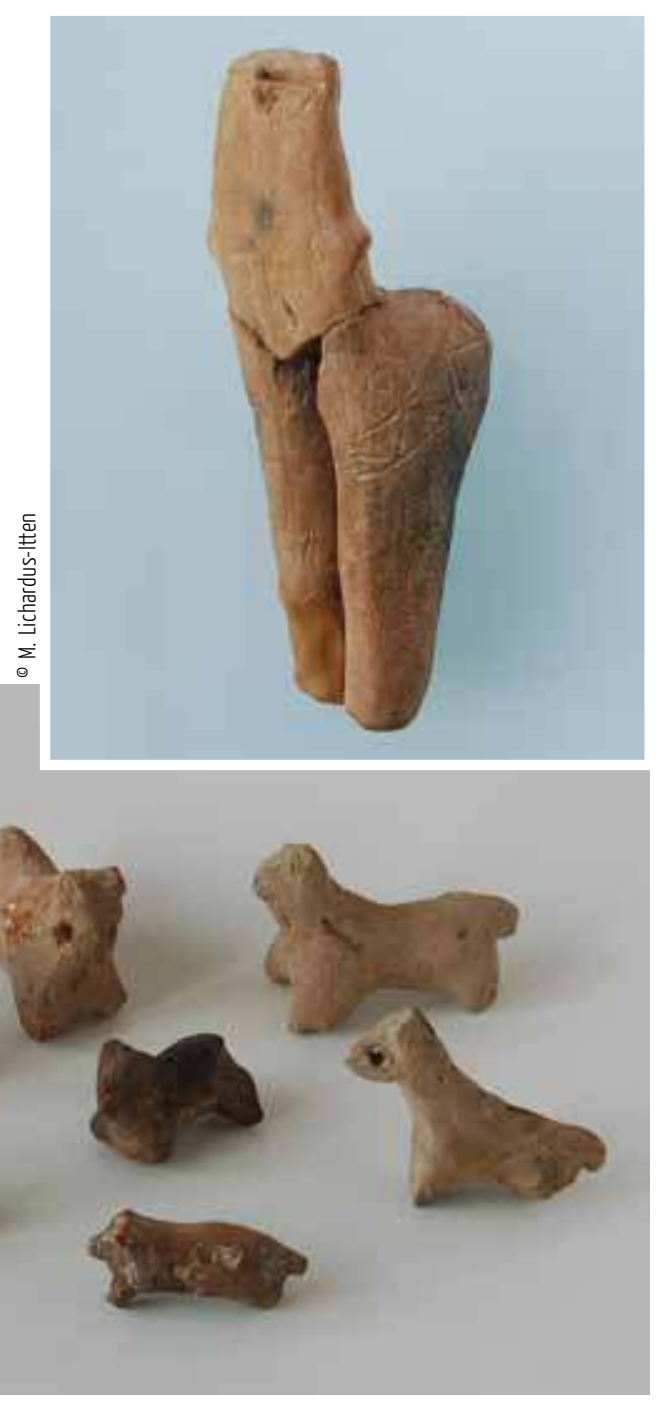




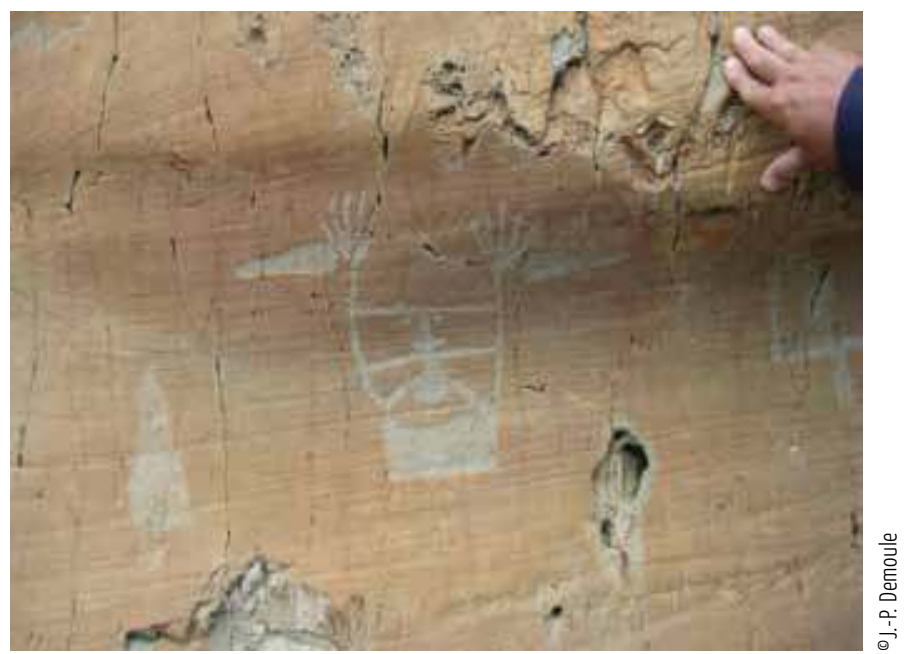

Gravure d'homme en armes, dite du " sorcier », sur l'un des rochers de la Vallée des Merveilles dans le parc du Mercantour, et datée du néolithique.

(Fig. 8)

Icône dans la tradition du christianisme orthodoxe, ici une copie récente d'un original plus ancien représentant Saint-Jean Baptiste.

(Fig. 9)

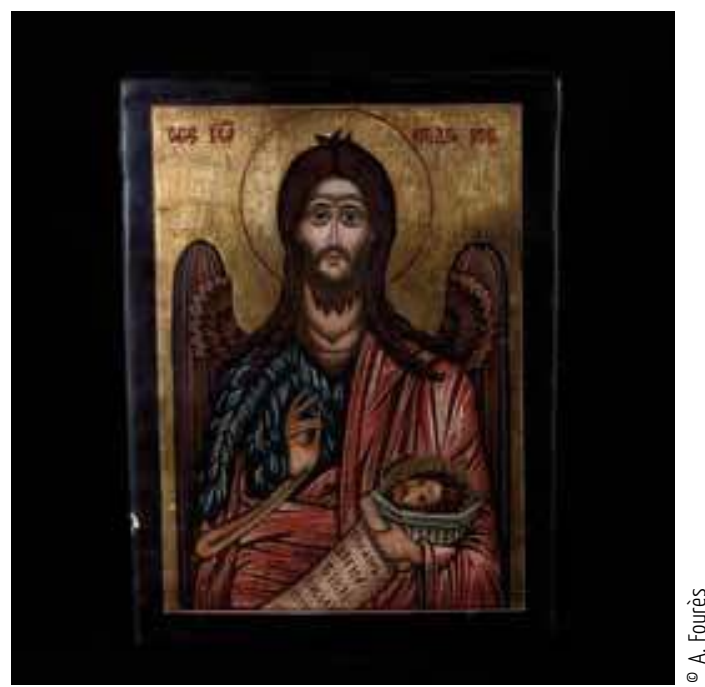

pouvoir, avec des hommes (mâles) en armes (Demoule 2007): Figure 8. Ces représentations ne feront que s'amplifier ensuite, durant tout l'âge du Bronze, avec non seulement des hommes en armes, mais des armes seules, des chevaux, des chars, des astres et, en Scandinavie, des bateaux. Les représentations solaires, ou plus généralement cosmiques, dont le disque d'or et de bronze trouvé à Nebra en Allemagne du nord est l'une des expressions les plus spectaculaires, sont caractéristiques de ces périodes. Globalement, la mise en scène de figures masculines de pouvoir, chefs, héros ou être surnaturels, semble bien coïncider avec l'émergence de chefferies de plus en plus complexes, comme le suggère le matériel archéologique. Durant toute cette trajectoire historique et sociale, on peut toutefois qualifier également d'art des objets utilitaires de facture soignée, qui vont depuis les « bâtons de commandement » en ivoire du paléolithique, jusqu'aux céramiques peintes néolithiques ou aux parures de l'âge du Bronze - distinction déjà mentionnée, entre un art du quotidien et un art que l'on peut qualifier de cérémoniel, et établie par Franz Boas dans son livre fondateur d'une anthropologie de l'art (Boas 1927; cf. aussi Layton 1981).

Avec l'apparition des premiers États, au Proche-Orient puis en Europe, une complexification croissante et une poussée démographique continue des formations sociales ouvrent de nouveaux supports et de nouvelles fonctions aux représentations. À côté de l'art politico-religieux, qui magnifie le pouvoir terrestre et céleste plus ou moins confondus (les pharaons sont d'ascendance divine et seule leur action permet que les crues bienfaisantes du Nil se reproduisent chaque année), et de l'art du quotidien, s'ouvre un nouvel espace pour les classes dirigeantes qui, sans exercer directement le pouvoir étatique, croissent en effectifs et jouissent d'un train de vie opulent. Ainsi les demeures princières ou «bourgeoises » des monarchies proche-orientales ou des principautés égéennes s'ornent de fresques, de statuettes, de bibelots décoratifs richement ornés, suscitant un artisanat spécialisé voué au luxe. Malgré la chute du monde minoen-mycénien et l'épisode des « âges sombres », cet art réapparaît bientôt dans le monde des cités helléniques et italiques et se perpétue, en s'amplifiant, dans l'Empire romain et sa culture gréco-romaine et en se diversifiant, au fur et à mesure que croît et que se complexifie la stratification sociale. Avec l'État, le modèle naturaliste s'impose dans les représentations humaines

Les remaniements de l'Antiquité tardive et du Haut Moyen Âge, avec la diffusion triomphante du christianisme, idéologie impériale par excellence, font passer au second plan pour un temps cet art « laïc », au bénéfice d'un art sacré, qui d'ailleurs fait beaucoup plus signe et symbole qu'il ne vise à représenter - le modèle naturaliste s'estompant en partie (Figure 9). La Renaissance et la redécouverte de l'Antiquité, et l'essor d'une bourgeoisie industrielle et marchande voient réapparaître, mais de plus en plus ample, le large spectre des formes et des fonctions artistiques de l'Antiquité gréco-romaine, tout comme le modèle naturaliste, que ce soit dans les représentations liées au sacré, ou au pouvoir, ou à l'art de prestige et d'agrément. 


\section{Reproduire le visible?}

Mais au cours du Xix siècle, une invention technique, celle de la photographie, bouleverse les fonctions usuelles des objets artistiques. Pour reprendre Paul Klee, l'art n'a plus à reproduire le visible, mais à rendre visible. Certes, les représentations du sacré et du pouvoir se perpétuent sous des formes traditionnelles, vastes tableaux peints et statues de pierre ou de bronze - plus encore avec l'art pompier et l'art sulpicien. Mais les portraits bourgeois peuvent désormais recourir à la photographie, laquelle, en se démocratisant, va se répandre dans presque toutes les couches de la société. Peu à peu, chacun pourra «faire » l'artiste, une activité qui s'est encore amplifiée avec la photographie numérique, pour atteindre une telle profusion qu'une grande partie des clichés ne sont même plus ni vus ni visibles, mais simplement stockés au fond de la mémoire des appareils, jusqu'à l'oubli ou l'effacement. Du coup, dans le courant du XIx ${ }^{e}$ siècle, avec Constable, Turner, puis les impressionnistes, peinture et sculpture sont peu à peu libérées de toute contrainte représentative - tout comme, un peu plus tard, poésie et littérature pourront jouer avec les mots et les sons au-delà de leur stricte fonction descriptive.

Cela n'affectera pas pour autant une partie des formes d'art traditionnelles. On continuera jusqu'à nos jours à dresser en pierre ou en bronze les portraits des grands dirigeants. On décorera les sanctuaires des diverses religions, du moins pour celles qui admettent les images, des représentations peintes ou sculptées de diverses entités divines. On ornera sa demeure, en fonction de ses moyens, d'œuvres d'art de valeur (changeante d'ailleurs, en fonction du «marché »), depuis les tableaux les plus coûteux jusqu'aux humbles bibelots ramenés comme souvenirs de voyages plus ou moins exotiques (Figure 10). Les élites intellectuelles baptisent actuellement kitsch ces objets de faible valeur marchande et produits en série à destination des classes pauvres : on tient donc là en creux et négativement l'une des définitions de l'«art». On maniera aussi cette « esthétique du quotidien » qui va des propulseurs sculptés du magdalénien jusqu'à ce que nous appelons le design.

Cette liberté nouvelle est bientôt explorée jusque dans ses plus extrêmes limites, au point que Marcel Duchamp peut poser de façon spectaculaire et provocatrice, avec ses ready made et la célèbre « fontaine », la question même de la définition de l'art. Celui-ci s'éloigne de la question du « beau », qui de Platon à Kant et Hegel n'avait cessé d'interroger les philosophes, pour s'affronter à l'acte ou à l'objet « gratuit», dont il devient en partie synonyme. De fait, la seconde partie du $\mathrm{xx}^{\mathrm{e}}$ siècle a assisté à une continuelle surenchère pour repousser les frontières de l'art, et de ce qui était considéré comme tel. N'importe quel objet, mais aussi n'importe quel acte, peut-être qualifié d'« artistique ». Déjà en 1958 Yves Klein avait créé l'événement avec son exposition « Le vide », à la galerie Iris Clert, où il n'y avait strictement rien d'exposé, mais seulement les cimaises peintes en blanc. Arman avait suivi deux ans plus tard en exposant « Le plein », c'est-à-dire en remplissant jusqu'au plafond la même galerie d'objets disparates et de rebuts.

Cette surenchère n'a plus jamais cessé. Et, de nos jours, on est parfois perplexe, sans forcer le trait (cf. Michaux 2004), dans une exposition d'art contemporain lorsque l'on se demande si, dans tel coin, telle chaise et tel extincteur font partie des œuvres d'art 


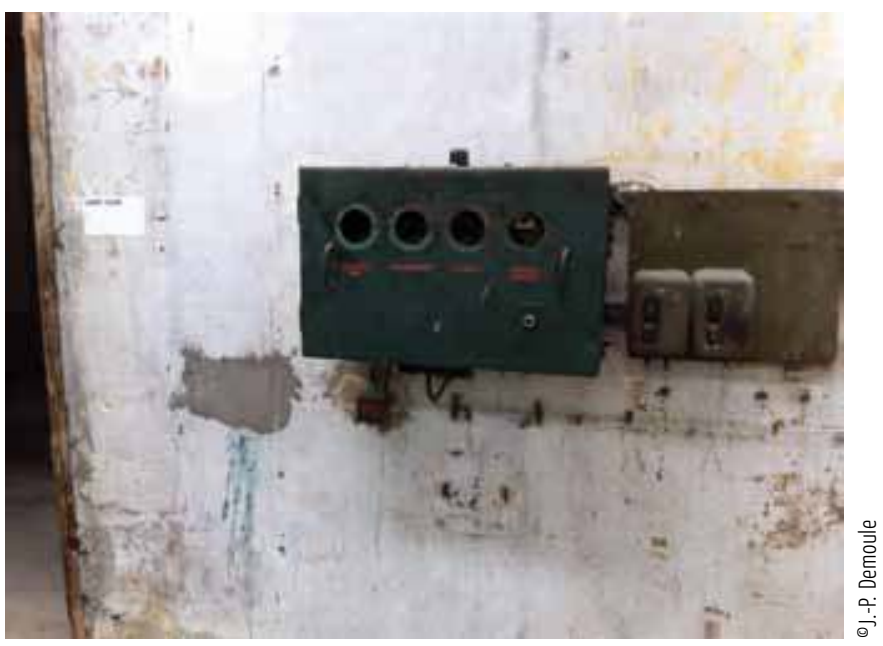

Boîtiers électriques dans l'un des hangars d'exposition des Rencontres Internationales de la Photographie en Arles, anciens ateliers de réparation de la SNCF : cet objet est-il un vestige ancien, ou la composante d'une installation artistique?

(Fig. 11) exposées, ou bien sont de simples accessoires (Figure 11). L'une des installations de l'artiste russe Ilia Kabakov fut justement une salle d'exposition délabrée où l'eau de pluie coulait dans des bassines. C'est finalement son lieu d'exposition, sinon son prix, qui décide du statut, artistique ou non, d'un objet, d'une composition, d'une performance. Et on peut trouver paradoxal que des ceuvres souvent explicitement critiques de la société contemporaine ne peuvent être acquises que par de riches collectionneurs, produits de ladite société inégalitaire et marchande, par l'intermédiaire de galeristes habiles, afin d'accroître encore leur prestige social - et leur fortune. Ainsi Jeff Koons qui fait fabriquer à l'échelle industrielle dans son atelier des objets en métal, mais copies d'objets de série en matière plastique (bouée de plage pour enfant par exemple), lesquels sont vendus comme de l'« art » à des prix exorbitants copies en matière durable d'objets éminemment fragiles et périssables, sinon remplaçables.

Telle est en tout cas la trajectoire, historique et anthropologique, que l'on peut proposer pour une histoire des images (Boas 1927; Schnapp 1997; Gell 1998; Belting 2004; Demoule 2007, 2011; Dufrêne \& Taylor 2009). Cette trajectoire est indissociable des sociétés concrètes qui ont produit ces images, et des fonctions sociales et idéologiques desdites images, même si l'anthropologie sociale, presque jusqu'à Levi-Strauss, Boas excepté, s'est peu intéressée aux images, tandis que l'archéologie ne les traitait que dans la perspective appauvrie d'une histoire de l'art classique.

\section{L'art et les déchets}

Le mouvement de remise en cause du statut de l'œuvre d'art initié par Marcel Duchamp, a longtemps été marginal. L'un de ses rares représentants dans l'entre-deux-guerres fut l'artiste allemand Kurt Schwitters, qui composa d'abord des collages d'objets ramassés dans la rue, avant de remplir intégralement sa maison d'une accumulation totale intitulée Merzbau, bientôt anéantie sous les bombardements américains puis reconstituée hors d'Allemagne. Il déclarait pour expliquer sa démarche et ce nouveau rapport aux objets ainsi institué:

« Je ne comprends pas pourquoi on ne pouvait utiliser dans un tableau, au même titre que les couleurs spécialement fabriquées pour les peintres, des matériaux tels que: vieux billets de tram ou de métro, morceaux de bois flotté, tickets de vestiaire, fil de fer, rayons de vélo, boutons, en un mot toutes les vieilleries qui traînent dans les greniers ou sur les tas d'ordures. C'était là, en quelque sorte, un point de vue social et, sur le plan artistique, un plaisir personnel...» (Schwitters 1994, 1999).

Cette intégration des débris n'est donc pas qu'esthétique. Elle s'inscrit dès Schwitters dans une problématique de la culture matérielle, de l'appréhension des objets d'une société. Minoritaire en ses débuts, cette réflexion s'amplifie après la seconde Guerre Mondiale. Non pas juste après, car devant les champs de ruines et les atrocités de ce conflit, la 
plupart des artistes d'avant-garde préfèrent s'exprimer dans l'abstraction. Mais à la fin des années cinquante, et surtout pendant les années soixante, des groupes d'artistes systématisent la réflexion sur les déchets que produisent, en nombre toujours croissant, les sociétés apparemment triomphantes des Trente Glorieuses. C'est ce qu'exprimeront des deux côtés de l'Atlantique des artistes comme les Nouveaux Réalistes en France, ou Rauschenberg et Kienholz aux États-Unis. Rauschenberg se revendique de Schwitters et écrit :

«Les objets que j'utilise sont la plupart du temps emprisonnés dans leur banalité ordinaire. Aucune recherche de rareté. À New York, il est impossible de marcher dans les rues sans voir un pneu, une boîte, un carton. Je ne fais que les prendre et les rendre à leur monde propre... ».

Plus radical, Kienholz monte des installations critiques pour dénoncer les aspects sordides de la société de consommation américaine. Son regard rejoint délibérément, sans le savoir, les démarches des archéologues. Il déclare à propos d'une exposition à Berlin Ouest intitulée Volksempfängers:

« Je ne commence réellement à comprendre une société qu'en parcourant ses brocantes et ses marchés aux puces. C'est une forme d'éducation et d'orientation historique pour moi. Je peux voir les résultats des idées par ce que jette une culture » (Kienholz $1977: 12$ )

En France, ce sont donc les Nouveaux Réalistes, regroupés en 1960 sous cette appellation par le critique d'art Pierre Restany (Figure 12), qui vont incarner ce travail sur les objets, bien que sous des formes très diverses (Debray 2007). Il n'y a à première vue pas grand-chose de commun entre les « anthropomorphes » d'Yves Klein, qui traîne sur des toiles blanches des femmes nues préalablement enduites de « bleu Klein » (renouant ainsi avec les mains positives des parois paléolithiques), les compressions de César, les emballages de Christo, les affiches déchirées de Villeglé, les fauteuils ou les pianos explosés ou les voitures récupérées d'Arman (Figure 13), ou les «tableaux-pièges » de Spoerri. Sinon que, Klein mis à part, ces artistes utilisent des objets en fin de vie pour en composer des « œuvres » et ont par là même tourné, entre autre, le dos à l'art abstrait.

En tant qu'ils récupèrent des objets mis hors du circuit de leurs fonctions usuelles, leur démarche est éminemment archéologique, pour autant que les archéologues ne fouillent jamais que des débris, villes détruites ou abandonnés, maisons en ruines et remblayées, objets jetés au rebut, etc. Même les tombes, si elles ne sont pas à proprement parler détritiques sont néanmoins une forme de mise à l'écart définitive. Les tableaux-pièges de Daniel Spoerri étaient néanmoins une mise à l'écart mise en scène. Il interrompait un repas en son milieu, figeait et fixait les assiettes, verres et couverts, et plaçait au mur le plateau de la table verticalement, comme un tableau. L'un d'eux porte en outre l'inscription « eaten by Duchamp », indication du rôle tutélaire du prestigieux convive. C'est toutefois parce que ces

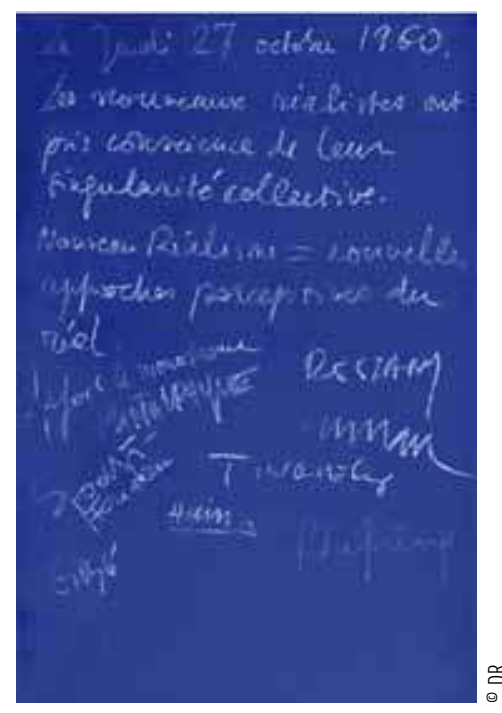

Texte original, daté du 27 octobre 1960, du Manifeste des Nouveaux Réalistes, rédigé par le critique d'art Pierre Restany et réunissant les signatures entre autres de Klein, Arman, Spoerri, Hains, Tinguely, Villeglé, etc. (Fig. 12)

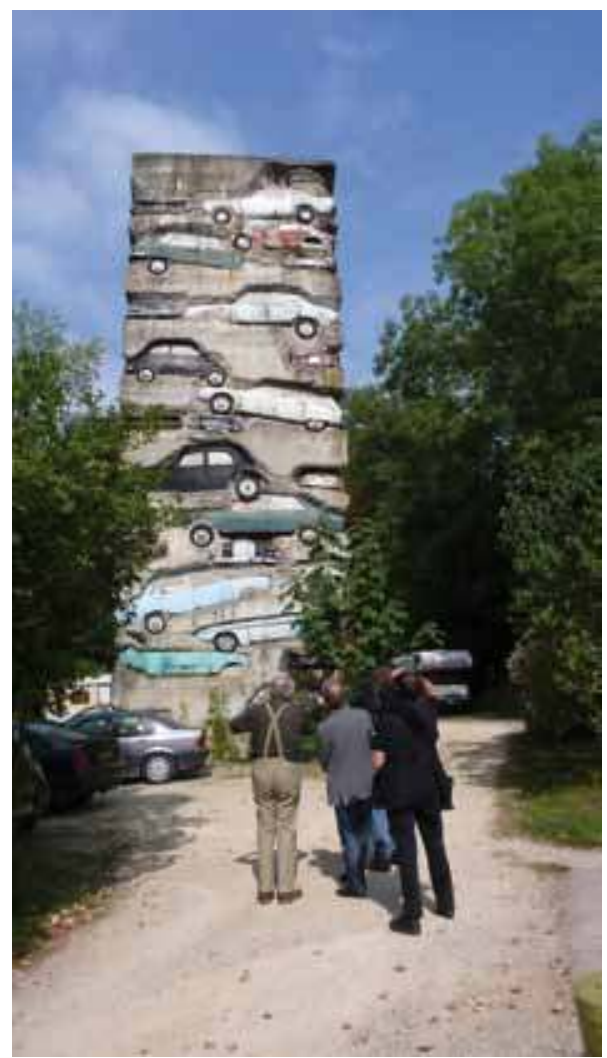


tableaux-pièges, qui sont estimés actuellement à environ 30000 euros pièce, tournaient au procédé, que Spoerri décida d'en accomplir un ultime, et en grand.

\section{Un Déjeuner sous l'herbe}

Le 23 avril 1983, il invita une centaine de convives des milieux « branchés » parisiens à un banquet de plein air dans le parc du château du Montcel, alors propriété du mécène Jean Hamon, et qui allait pour un temps, à partir de l'année suivante, devenir le siège de la Fondation Cartier. Au milieu du repas, lequel s'interrompt, les plateaux des tables, qui reposaient sur des tréteaux, sont emmenés avec tout ce qu'ils contiennent et déposés au fond d'une grande tranchée préalablement creusée à proximité. Puis l'on recouvre de terre ce nouveau tableau-piège (desserts et cafés sont servis un peu plus loin), et l'herbe repousse à cet endroit - d'où le nom de Déjeuner sous l'herbe que donna Daniel Spoerri à la performance, en référence à Manet - et à une longue tradition picturale de déjeuners champêtres. À l'origine, un archéologue ami de Daniel Spoerri, Éric Godet, aurait dû fouiller quelques années plus tard l'œuvre, qui était donc explicitement archéologique, mais qui avait, polysémique comme tout travail fort, plusieurs significations. Elle aurait aussi résulté d'un pari avec le peintre allemand Konrad Klapeck, pari dont l'enjeu était de savoir quelle production des deux artistes aurait la plus grande longévité; mais on ne sait pas si Klapeck enterra l'une de ses toiles, qui serait aujourd'hui dans un bien piètre état. Elle pouvait évoquer également la tranchée devant laquelle aurait été fusillé, en présence de son jeune fils, le père de Daniel Spoerri, juif roumain, par les Einsatzgruppen nazis durant la dernière guerre. Bernard Müller, anthropologue qui me contacta en 2009 pour réaliser la fouille, a supposé aussi que ce happening collectif aurait été pour ces

Fouille en cours du Déjeuner sous l'Herbe.

(Fig. 14)

Le Déjeuner sous I'Herbe, vue de la partie qui a été moulée en résine et en bronze.

(Fig. 15)

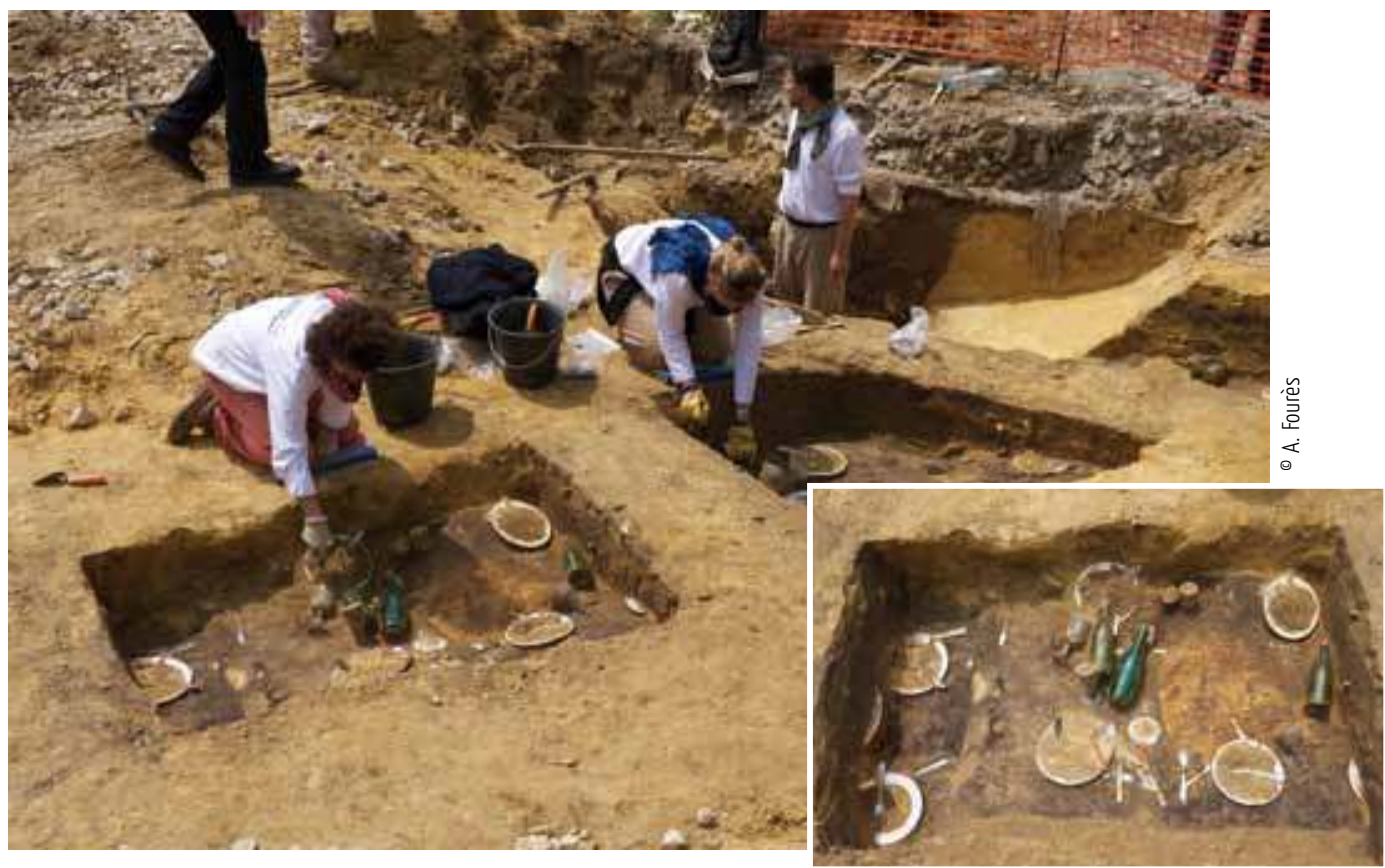


intellectuels en vogue une façon d'enterrer leurs illusions avec le tournant de la rigueur qui suivit de deux ans la victoire de François Mitterrand à l'élection présidentielle de 1981 (Müller 2011).

Toujours est-il que ces «premières fouilles de l'art moderne » ne se firent pas, Éric Godet étant entré entretemps dans les ordres, ce que commenta ainsi Daniel Spoerri: « quand on trouve Dieu, on perd les assiettes »... Lanthropologue Bernard Müller eut vent de ce chantier en déshérence et me contacta. La fouille fut donc organisée en 2010, avec tout le protocole d'une fouille archéologique rigoureuse et avec l'appui de l'Institut national de recherches archéologiques préventives (Figures 14-15; Demoule à paraître) ${ }^{1}$. Toute fouille a un coût, celui du salaire des archéologues professionnels, ainsi que les frais matériels et d'étude. Notons que ces frais, pris en charge par l'Inrap (à l'exception de mon salaire, qui relevait de mon Université), ne furent pas imputés sur la ligne budgétaire « recherche » de cet institut, mais sur sa ligne « développement culturel et communication ». Il fut estimé en effet par les responsables de l'Inrap, dont je n'étais déjà plus président à l'époque, que les retombées attendues de l'opération seraient plus en termes de « communication » que de « recherche». Ou plus exactement, on pouvait craindre de la part de la tutelle ministérielle, souvent malveillante, de l'Inrap, mais aussi de la part de certains de ses archéologues, qu'il soit estimé qu'on manquait suffisamment de moyens pour les « choses sérieuses", pour ne pas les disperser sur des opérations discutables. Alors que, avec raison, nul ne pouvait contester que la fouille du Déjeuner sous l'herbe serait profitable à l'Inrap en termes de communication - ce qui fut effectivement le cas, avec une cinquantaine de relais dans des médias nationaux ou internationaux, dont aucun ne remit d'ailleurs en cause l'intérêt de la fouille.

Plusieurs laboratoires de sciences naturelles y furent également associés, intéressés à des recherches expérimentales sur des objets enfouis dans des conditions contrôlées. La fouille eut aussi un intérêt épistémologique, en montrant les écarts qui existaient déjà entre les témoignages oraux et écrits sur l'événement (matière normale des historiens), et ce que retrouvaient les archéologues, divergences qui concernaient par exemple l'emplacement exact de la tranchée, la matière des plateaux des tables ou encore l'existence (ou non) de couverts en plastique, voire en carton. L'archéologie n'était définitivement pas « une discipline auxiliaire de l'histoire », selon l'arrogante définition classique des historiens.

Un autre intérêt épistémologique était de savoir si tout cela était bien de l'archéologie. En effet, j'avais déposé auprès du ministère de la Culture une demande d'autorisation de fouille en bonne et due forme. Elle me fut refusée (Figure 16) dans un long avis qui se terminait ainsi: « La commission considère que cette demande de fouille programmée, par son objet, ne relève pas de la recherche archéologique telle que définie dans le code du patrimoine », précisant même: "Quant à un débat sur la nature de l'archéologie et ses limites, il est de fait faussé par la personnalité même du porteur du projet »... Cet avis n'interdisait pas que l'on creuse dans le parc du château du Montcel, mais signifiait seulement 


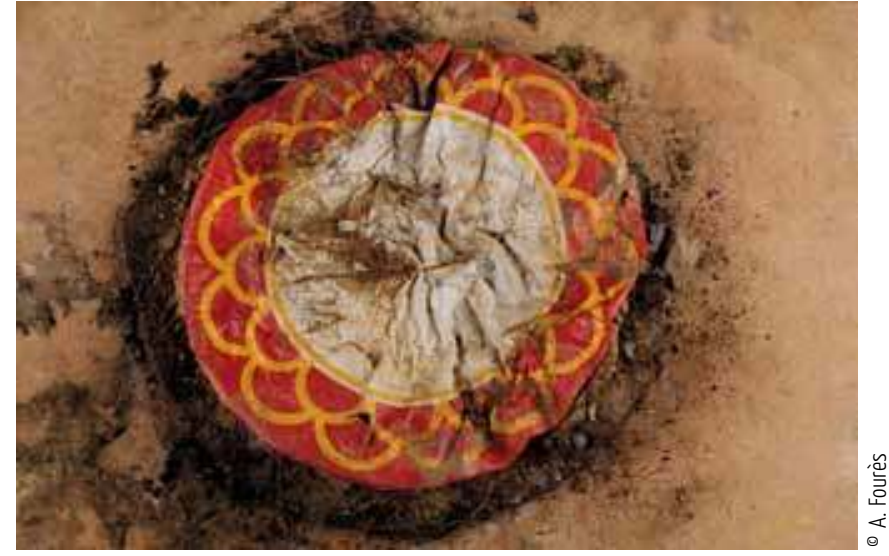

Assiettes appartenant au Déjeuner sous l'Herbe et exhumées lors de la fouille de juin 2010: art, ou pas art?

(Fig. 17) que ce ne serait pas un acte archéologique. Pourtant le «Code du Patrimoine » ainsi appelé en renfort stipule dans son article L. 510-1: « Constituent des éléments du patrimoine archéologique tous les vestiges et autres traces de l'existence de l'humanité, dont la sauvegarde et l'étude, notamment par des fouilles ou des découvertes, permettent de retracer le développement de l'histoire de l'humanité et de sa relation avec l'environnement naturel ». Ce qui évidemment ne fixe aucune date de " fin de l'archéologie », d'autant que l'archéologie du temps présent est depuis un certain temps, aux ÉtatsUnis mais aussi en France, une discipline bien admise et a fait l'objet de plusieurs colloques et travaux collectifs (Schnapp 1997; Demoule \& Stiegler 2008; Mientjes 2009; Journot \& Bellan 2011).

Le ministre de la Culture de l'époque, Frédéric Mitterrand, devait se rendre sur la fouille à l'occasion des « Journées nationales de l'archéologie », qui coïncidaient avec leur achèvement. La fouille réunissait en effet deux activités, la création artistique et la recherche archéologique, dont ce ministère est comptable. Toutefois, au vu du refus par ses services de considérer la fouille comme relevant de l'archéologie, le malheureux ministre fut vigoureusement dissuadé par lesdits services, dont l'honneur était ainsi mis en jeu, de se rendre sur le Déjeuner sous l'herbe. Comme quoi d'insignifiantes assiettes peuvent devenir une affaire d'État quand le pouvoir, ou plutôt son simulacre, est en jeu...

\section{Irremplaçables}

Mais un autre aspect du Déjeuner touche directement à l'irremplaçabilité des objets (Figure 17). Les convives, en effet, devaient apporter leurs propres couverts, ainsi qu'un objet personnel, sachant que l'ensemble serait enterré et qu'ils n'en disposeraient donc plus. Niki de Saint-Phalle en particulier, d'après les témoignages, avait façonné quelques objets décoratifs pour orner la table. L'intérêt de la fouille a été de montrer que les couverts abandonnés étaient de très bas prix, assiettes et verres de cantine, voire de pique-nique, gobelets en plastique - en dépit des souvenirs de Daniel Spoerri. Ce fut même l'une des surprises d'une critique d'art, Anne Tronche, spécialiste en particulier du mouvement des Nouveaux Réalistes et coréalisatrice de l'un des films sur Spoerri, qui, bien qu'invitée, n'avait pu assister au Déjeuner sous l'herbe, de découvrir au moment de la fouille la faible valeur des objets déposés. Elle le vécut, à trente ans de distance, comme une forme d'offense faite à l'artiste de par la pingrerie des invités. Certes, les objets n'étaient pas voués à un abandon définitif, puisque leur fouille avait été prévue dès l'origine, mais il était peu probable que leurs propriétaires en revendiquent jamais la restitution.

Outre les couverts, ce fut aussi le cas des objets personnels. Si les éléments totalement périssables avaient disparu (des fleurs ornaient les tables, d'après les 
photos de l'époque), nous retrouvâmes quelques pièces de monnaie ne dépassant pas la valeur faciale de 10 centimes de l'époque - les monnaies sont néanmoins un précieux moyen de datation archéologique, dit post quem. Plus personnelle, une brosse à dent (usagée) de couleur rouge au manche en forme de femme nue, comme il s'en trouvait dans les salles de bain durant les années 1970-1980. Elle fut baptisée « Venus de Jouy » (Figure 18). Peut-être passerait-elle pour un objet rituel aux yeux d'archéologues du futur, puisque telle est en général la fonction que nous attribuons aux découvertes dont l'usage utilitaire nous échappe. Elle était déposée dans une assiette, à côté des couverts. Elle appartient en tout cas à cette longue filiation de l'« art du quotidien » issu du paléolithique et rebaptisé design.

En résumé, ce que l'élite artistique et intellectuelle branchée des années 1980 avait jugé bon d'abandonner, irremplaçablement, sous la terre et l'herbe du domaine du Montcel n'avait guère de valeur marchande.

Ils ne sont pas pour autant devenus des objets d'art. En effet, la question se posa lorsque se tint au musée d'art Pasquart de Bienne en Suisse la première exposition explicitement consacrée à « Archéologie et art contemporain» (Denaro 2011). Comme il fallait bien assurer les objets, la question de leur statut fut posée à Daniel Spoerri. Il répondit par lettre: « Les objets exhumés et exposés en dehors de leur contexte initial de performance artistique ne peuvent être considérés comme l'œuvre originale et doivent être simplement considérés comme des archives documentant la performance originelle ». S'ils ne pouvaient donc être vendus comme objets d'art, ils furent néanmoins assurés, car c'était obligatoire, sur la base de la vente d'un tableaupiège de Daniel Spoerri datant de 1985 et vendu 32000 euros en 2010 chez Christie's. En outre, puisque le Déjeuner sous l'Herbe fut exposé à Bienne, dans un musée d'art et à la demande de ses conservateurs, parmi les œuvres d'une vingtaine d'autres artistes, il appartient donc bien à l'art (Figure 19).

Toute fouille étant une destruction, s'est posé aussi le problème de l'enregistrement archéologique du Déjeuner sous l'Herbe. Aussi, outre les relevés traditionnels au crayon et par photographie, ont été réalisés une couverture photo en trois dimensions; un scannage, également en trois dimensions (Photo d'ouverture - Figure 20), lequel permet à son tour des tirages tridimensionnels à des échelles et dans des matières variées; et enfin un moulage au latex, qui a son tour a permis d'une part un tirage en résine, et trois tirages en bronze, sous l'égide de Daniels Spoerri,

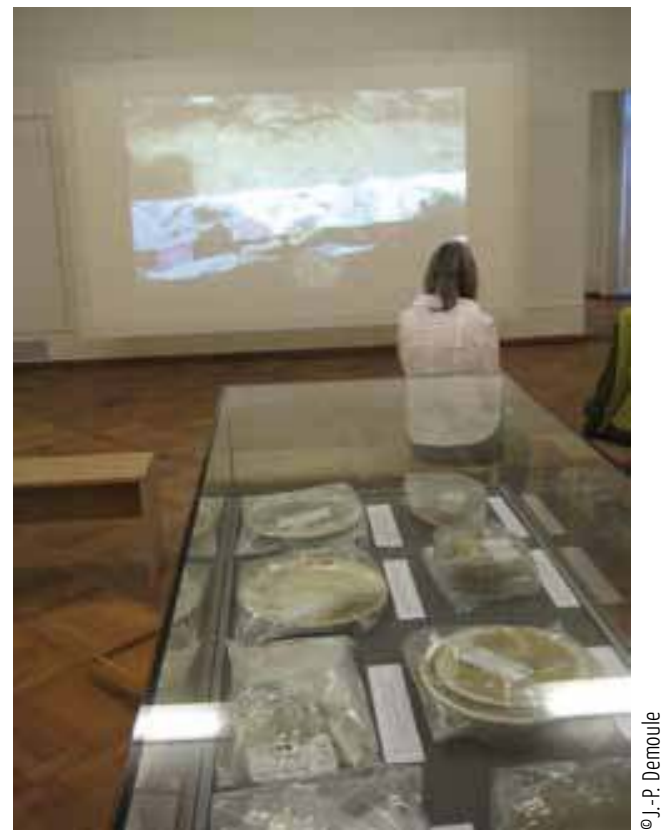

Le Déjeuner sous I'Herbe exposé au Centre Pasquart à Bienne en Suisse, première exposition internationale (Arkheologia) consacrée, à l'automne 2009, sur $1600 \mathrm{~m} 2$ à la rencontre entre archéologie et art contemporain.

(Fig. 19)

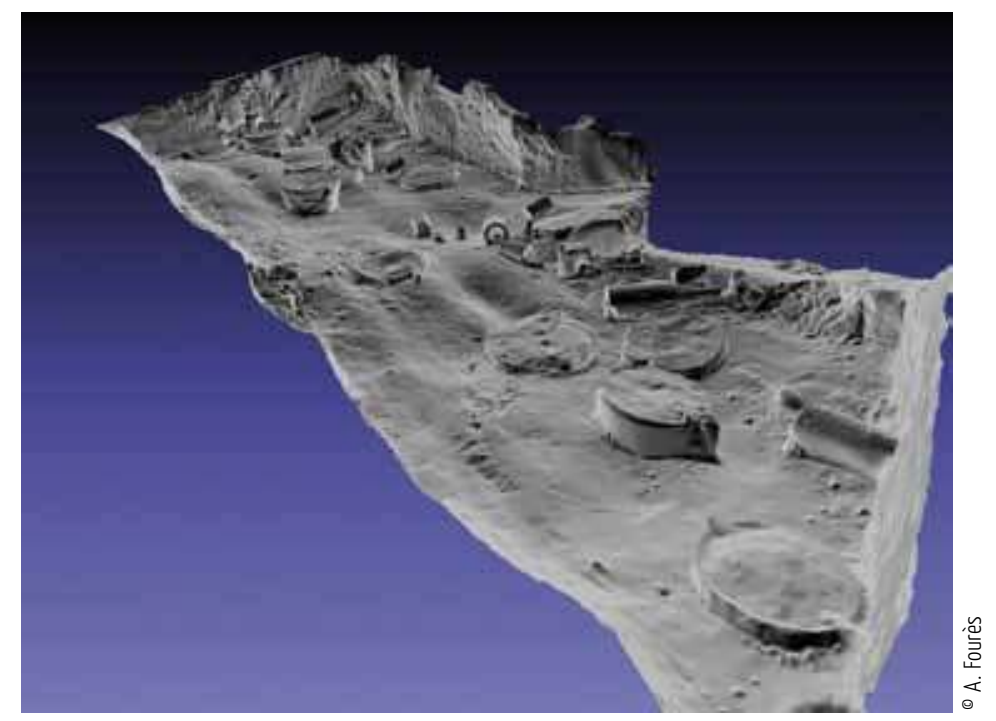

Enregistrement 3D du Déjeuner sous l'Herbe, permettant de produire des maquettes en $3 \mathrm{D}$ à différentes échelles (Veodis 3D).

(Fig. 20) 


\section{À quoi sert l’art}

Comme le montre l'exposition de Bienne, qui regroupait les œuvres d'une trentaine d'artistes, la rencontre de l'archéologie et de l'art contemporain était bien dans l'air et s'était amorcée dès le début des années 2000 en Angleterre, pour se développer progressivement (Renfrew 2003, Gosden et al. 2009, Djaoui 2009, Jasmin \& Norcia à paraître, Pinchon \& Plana-Mallart 2012). Cette rencontre est aussi un symptôme. De deux manières différentes, nos sociétés contemporaines s'interrogent sur la trajectoire des sociétés et sur leurs vestiges, et finalement sur l'irremplaçabilité, sur ce qui ne sera plus jamais, mais qui a pourtant laissé des traces. Ce fut aussi dès l'origine l'une des fonctions de l'art. En laissant l'empreinte colorée de leurs mains sur les parois minérales des grottes, les hommes préhistoriques d'il y a 25000 ans pensaient aussi échapper à l'usure du temps, ce qu'ils ont d'ailleurs réussi. Lorsque le régime coréen érige au cœur de Pyongyang les deux gigantesques statues de bronze, hautes de vingt mètres, des deux grands dirigeants Kim Il-sung et de Kim Jong-Il, il pense bien s'inscrire dans l'éternité. Et cette prétention n'est pas propre aux régimes totalitaires, puisqu'une statue de bronze a été récemment érigée sur les Champs Élysées, montrant le Général de Gaulle arpentant l'espace d'un pas décidé. Sans compter le défunt Georges Frèche, maire de Montpellier, qui a lancé le projet, désormais réalisé, de douze statues en bronze, chacune de trois mètres de hauteur, érigées avec une certaine dose usuelle de provocation sur la place publique dite « des Grands Hommes » du nouveau quartier Odysseum, et représentant entre autres le même général de Gaulle, mais aussi Churchill, Jaurès, Roosevelt, Gandhi, Lénine et Mao Tsé Toung (Figure 22).

Les fonctions de l'art, c'est une banalité, sont multiples: re-présenter ce qui n'est pas présent, échapper au défi du temps, afficher son statut social, rendre plus plaisants certains actes de la vie quotidienne (ce qu'on appelle le design), avoir prise sur le surnaturel supposé, réassurer la cohérence d'un groupe social, etc. Pour ne pas parler de la définition du « beau », sur laquelle ne cesse de s'interroger une longue tradition philosophique. Que l'art contemporain transforme en un objet par définition irremplaçable - l'œuvre d'art - une accumulation d'objets volontairement abandonnés, donc remplacés, est certainement l'un des signes éclatants que les sociétés humaines actuelles, du moins celles qui produisent et manipulent de tels objets, sont d'une nature nouvelle.

Un peu partout se multiplient ce qu'on appelle des « capsules temporelles», au point qu'une université américaine, celle d'Oglethorpe à Atlanta aux ÉtatsUnis, accueille la International Time Capsule Society, laquelle recense toutes ces expériences où l'on enfouit dans le sol des objets divers, afin de créer des liens, au-delà même de notre propre et irremplaçable disparition, avec les humains du futur. Parmi les expériences spatio-temporelles les plus connues, citons celles des sondes spatiales Pioneer X et XI et Voyager 1 et 2, qui depuis les années 1970 transportent de plus en plus loin de la Terre une concentration des connaissances humaines gravées sur des plaques de métal. À Nantes, l'opération « Le grenier du siècle » a scellé dans un mur de l'ancienne usine Lu, devenu lieu culturel, 16000 boîtes métalliques contenant des objets personnels

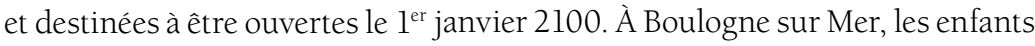
d'un centre éducatif ont réalisé en 2010 un "Petit-déjeuner sous l'herbe ",
Georges Frèche, maire de Montpellier, inaugurant la statue en bronze de Lénine destinée à la « Place des Grands Hommes » du quartier Odysseum.

(Fig. 22)

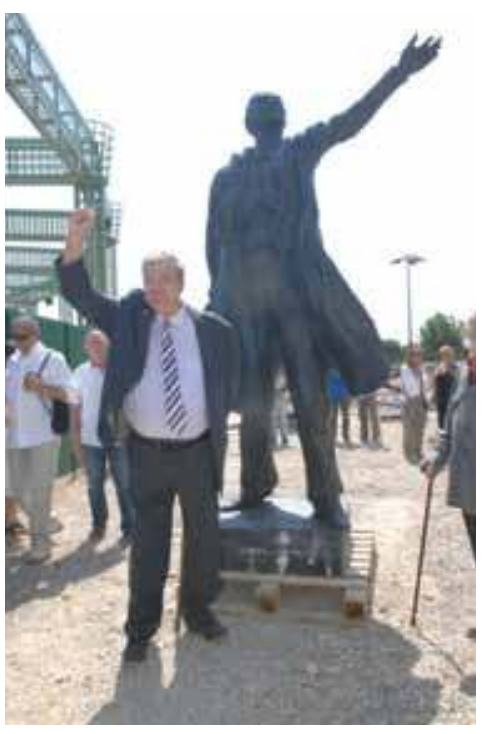


en référence à la fouille du Déjeuner sous l'herbe, par l'enfouissement pour vingt ans d'objets personnels - et l'on sait la proximité métaphorique qu'entretiennent dès l'origine l'archéologie et la psychanalyse (Demoule 2003). En 2030, si l'archéologie en a alors les moyens techniques et en fonction de la taphonomie, sans doute retrouvera-t-on parmi les objets précieusement déposés les restes d'un nounours en peluche, comme celui qui orne la couverture de ce numéro.

Jusqu'à preuve du contraire, la mort, la disparition, la finitude, restent la seule limite qui s'impose irrémédiablement à chaque humain. Il n'est pas surprenant que depuis au moins trente mille ans la performance artistique soit sous des formes multiples et évolutives l'un des moyens de tenter d'échapper à cette évidence implacable. 


\section{NOTES}

Photo d'ouverture: La brosse à dent, dite désormais Venus du Jouy, exhumée lors de la fouille du Déjeuner sous l'Herbe et faisant partie des « objets personnels » volontairement abandonnés sur la table du banquet.

1. La fouille eut lieu du 31 mai au 7 juin 2010. Y participèrent directement, du côté de l'Inrap, Aurélia Alligri, Michel Baillieu, Mehdi Belarbi, Sandrine Henry-Duplessis, Nathalie Karst, Mercedes PionMaya, François Renel. La couverture photographique fut assurée par Anne Fourès et Denis Glicksman. Un moulage d'une partie de la fouille fut réalisé par Mehdi Belarbi, sur les conseils de Pascal Raymond, ainsi qu'une couverture photographique $3 \mathrm{D}$, tandis qu'un enregistrement par scanner 3D fut exécuté par Stéphane Petit (Université de Clermont-Ferrand). Le moulage fut ensuite tiré en bronze en trois exemplaires par les soins de Daniel Spoerri. La large médiatisation fut coordonnée par Paul Salmona, Vincent Charpentier et Mahaut Tyrrell (Inrap), et la fouille fit partie de celles présentées au grand public lors de la journée de l'archéologie Inrap/Arte du 5 juin 2010. Un film a été tourné en continu par Laurent Védrine: « Le Déjeuner sous l'herbe », Tingo Film, 52'. Plusieurs laboratoires et chercheurs ont mené ensuite les analyses post-fouilles, dont Martine Regert (CNRS, Cepam, Nice), Stéphanie Thiébault et Véronique Matterne (CNRS UMR 7209), Julia Wattez et Muriel Boulen (Inrap), Olivier Weller (CNRS, Arscan), Armelle Charrié (Institut de Chimie, CNRS et Université de Strasbourg). Plus de précision sur la fouille avec les liens: [http://www.inrap.fr/ archeologie-preventive/Actualites/Communiques-depresse/Les-derniers-communiques/Communiquesnationaux/p-11884-Fouille-archeologique-duDejeuner-sous-1-herbe-de-.htm] et: [http://www. sdtp.eu/] Consultés le 8 juillet 2012

\section{RÉFÉRENCES}

Belting H. 2004 Pour une anthropologie des images. Paris: Gallimard.

Benjamin W. 2000 L'œuvre d'art à l'époque de sa reproductibilité technique (dernière version 1939). In « Euvres III ». Paris: Gallimard.

Boas F. [1927] Primitive art. Traduit de l'anglais par M. Mauzé 2003 L’Art primitif. Paris: Adam Biro.

Debray C. (Dir.) 2007 Le Nouveau Réalisme. Paris: Réunion des Musées Nationaux

Demoule J.-P. 2003 Les Pierres et les mots, Freud et les archéologues. Alliage (52): 29-144 (republié in: Grands Articles (2) 2006).

— 2007 Naissance de la figure, du paléolithique à l'âge du Fer. Paris: Éditions Hazan.

- 2011 Images des archéologues et archéologie des images. In: Ph. Boissinot (Dir.) L’archéologie comme discipline? Paris: Éditions du Seuil (Le Genre Humain, n 50), 31-54.

- (à paraître) «Archéologie, déchets et art contemporain: le mémoriel et le matériel ». In: M. Jasmin \& A. Norcia, Des temps qui se regardent. Dialogue entre art contemporain et archéologie. Actes du colloque de l'INHA, 5-6 octobre 2009. 
Demoule J.-P. \& Stiegler B. 2008. L’Avenir du passé, modernité de l'archéologie. Paris: Éditions de la Découverte et Inrap.

Denaro D. 2011 Arkhaiologia. L'archéologie dans l'art contemporain. Nürnberg: Verlag für moderne Kunst

Djaoui D. (dir.) 2009 Lab Book, Marc Dion. Carnet de fouilles, Luc Long. Arles: Éditions Actes Sud et Musée Départemental Arles Antiques.

Dufrêne Th. \& Taylor A.-Chr (Dir.) 2009 Cannibalismes disciplinaires. Quand l'histoire de l'art et l'anthropologie se rencontrent. Paris: INHA et Musée du Quai Branly.

Gell A. 1998 Art and agency: an anthropological theory. Oxford: Clarendon Press; traduction française, 2009 L'art et ses agents - une théorie anthropologique. Paris: Les Presses du réel.

Gosden Chr., DeMarrais E. \& Renfrew C. 2004 Substance, memory, display. Archaeology and art. McDonald Cambridge: Institute for Archaeological Research.

Jasmin M. \& Norcia A. (à paraître) Des temps qui se regardent. Dialogue entre art contemporain et archéologie. Actes du colloque de l'INHA, 5-6 octobre 2009

Journot J. \& Bellan G. (Dir.) 2011 Archéologie de la France moderne et contemporaine. Paris: La Découverte/ Inrap

Kienholz E. 1977 Volksempfängers. In J. Merkert (Dir.) Berlin: Nationalgalerie.

Layton R. 1981 The Anthropology of art. London: Granada Publishing (Paul Elek).

Michaux Y. 2004 L'art à l'état gazeux. Paris: Hachette.

Mientjes A. C. 2009 Une archéologie du monde moderne. In J.-P. Demoule (Dir.) L’Europe, un continent redécouvert par l'archéologie. Paris : Gallimard-I.N.R.A.P.

Müller B. 2011 Festin-piège pour utopies mortes - Ethnographie du "Déjeuner sous l'herbe » à l'occasion de l'enterrement du tableau-piège (23 avril 1983 - juin 2010). Montréal: Musée des beaux-arts de Montréal/ Éditions Parachute.

Pinchon J.-F. \& Plana-Mallart R. (Dir.) 2012 Le Temps des Styrènes. Nîmes, Montpellier: Presses universitaires de la Méditerranée/École Supérieure des Beaux-Arts de Nîmes (Actes du colloque de Lattes et Montpellier, novembre 2011).

Renfrew C. 2003 Figuring it out. London: Thames \& Hudson.

Schnapp A. 1997 Le chasseur et la cité. Chasse et érotique dans la Grèce Ancienne. Paris: Albin Michel.

Schnapp A. (Dir.) 1997 Une archéologie du passé récent. Paris: Fondation Maison des sciences de l'homme Schwitters K. 1994 I, Manifestes théoriques et poétiques. Paris: Éditions Ivrea.

— 1999 Le point sur le i. Paris: Centre Georges Pompidou (LAart en jeu). 


\section{RÉSUMÉ}

Archéologie, art contemporain et recyclage des déchets. Les œuvres d'art sont par nature des objets éminemment irremplaçables. Toutefois ce terme recouvre des réalisations dont la fonction a été très diverse: centrée sur la sexualité au paléolithique; célébrant le pouvoir et son allié le divin à partir du chalcolithique; illustrant le prestige des élites dans les sociétés urbaines; et aussi, conférant une dimension esthétique à des objets utilitaires (ce que nous appelons aujourd'hui le design). Avec l'invention de la photographie, l'art de nos sociétés industrielles contemporaines n'a plus eu à reproduire et n'a donc cessé de s'interroger sur ses nouvelles fonctions. Ainsi se sont développés l'art abstrait et, en liaison avec leur abondance croissante, le recyclage des déchets sous forme d'œuvres d'art, transformant en objets irremplaçables des objets remplaçables et remplacés. Le cas du Déjeuner sous l'Herbe de Daniel Spoerri, «premières fouilles archéologiques de l'art moderne », est ici développé. L'archéologie, indissociable de l'histoire de l'art en ses débuts, retrouve aujourd'hui, en tant qu'étude des sociétés humaines à travers leurs déchets, les préoccupations de certains courants artistiques (Nouveaux Réalistes, etc.), qui réfléchissent sur la durabilité des sociétés humaines et sur ce qu'il en restera.

\section{ABSTRACT}

Archeology, contemporary art and recycling waste. Works of art are by nature eminently irreplaceable objects. Yet the term covers works whose functions have been very diverse and have become superimposed through time: focussed on sexuality in the Palaeolithic; celebrating power and its ally the divine from the Chalcolithic onwards; illustrating the prestige of elites in urban societies; and also, conferring an aesthetic dimension to utilitarian objects (what we now call design). With the invention of photography, the art of our present-day industrial societies no longer has to reproduce reality and is thus continuously wondering about its new functions. So abstract art developed and, linked to their increasing quantity, the recycling of refuse as works of art, transforming replaceable and replaced objects into irreplaceable objects. The case of the Déjeuner sous l'Herbe by Daniel Spoerri, the « first archaeological excavation of modern art », is discussed here. Archaeology, in its early days inseparable from art history, now rediscovers, as the study of human societies through their refuse, the preoccupations of certain artistic movements (Nouveaux Réalistes, etc) which are thinking about the durability of human societies and what will remain of them.

\section{MOTS-CLÉS}

Archéologie, art, histoire de l'art, déchets, réalisme, photographie.

\section{KEYWORDS}

Archaeology, art, art history, garbage, realism, photography. 